\title{
Solid State Fermentation of Brewers' Spent Grains for Improved Nutritional Profile Using Bacillus subtilis WX-17
}

\author{
Yong Xing Tan ${ }^{1,2,+}$, Wai Kit Mok ${ }^{3, \dagger}$, Jaslyn Lee ${ }^{3}$, Jaejung Kim ${ }^{3 \oplus}$ and Wei Ning Chen ${ }^{3, * \mathbb{C}}$ \\ 1 Interdisciplinary Graduate School, Nanyang Technological University, 50 Nanyang Avenue, \\ Singapore 639798, Singapore \\ 2 Advanced Environmental Biotechnology Centre, Nanyang Environment and Water Research Institute, \\ Nanyang Technological University, 1 CleanTech Loop, 1 CleanTech One \#06-08, Singapore 637141, Singapore \\ 3 School of Chemical and Biomedical Engineering, Nanyang Technological University, 62 Nanyang Drive, \\ Singapore 637459, Singapore \\ * Correspondence: WNChen@ntu.edu.sg; Tel.: +65-6316-2870 \\ + These authors have equally contributed to this work.
}

Received: 6 May 2019; Accepted: 15 June 2019; Published: 27 June 2019

\begin{abstract}
Brewers' spent grains (BSG) are underutilized food waste materials produced in large quantities from the brewing industry. In this study, solid state fermentation of BSG using Bacillus subtilis WX-17 was carried out to improve the nutritional value of BSG. Fermenting BSG with the strain WX-17, isolated from commercial natto, significantly enhanced the nutritional content in BSG compared to unfermented BSG, as determined by the marked difference in the level of metabolites. In total, 35 metabolites showed significant difference, which could be categorized into amino acids, fatty acids, carbohydrates, and tricarboxylic acid cycle intermediates. Pathway analysis revealed that glycolysis was upregulated, as indicated by the drop in the level of carbohydrate compounds. This shifted the metabolic flux particularly towards the amino acid pathway, leading to a 2-fold increase in the total amount of amino acid from $0.859 \pm 0.05$ to $1.894 \pm 0.1 \mathrm{mg}$ per $\mathrm{g}$ of BSG after fermentation. Also, the total amount of unsaturated fatty acid increased by 1.7 times and the total antioxidant quantity remarkably increased by 5.8 times after fermentation. This study demonstrates that novel fermentation processes can value-add food by-products, and valorized food waste could potentially be used for food-related applications. In addition, the study revealed the metabolic changes and mechanisms behind the microbial solid state fermentation of BSG.
\end{abstract}

Keywords: solid state fermentation; brewers spent grain; metabolomics; nutrition; pathway analysis

\section{Introduction}

An estimated one third of the food produced globally is lost during processing or wasted. Global food production is expected to rise due to the expanding population in the world. This would lead to huge amounts of food wastages annually, which cause significant environmental, economic, and climate issues. The nutritional contents remaining in these food processing residues can be harnessed to potentially become materials for other processes. Utilizing these residues would be a viable and cost-effective solution to the issues caused by massive amounts of food wastes generated worldwide [1].

In the beer industry, large quantities of by-products are generated. In particular, brewers' spent grain (BSG) represents $85 \%$ of the total by-products [2]. The remaining by-products includes spent hops and surplus yeast. The annual global production of BSG is estimated to be $38.6 \times 10^{6}$ tons [3]. It was reported that approximately $14-20 \mathrm{~kg}$ of BSG could be generated from $100 \mathrm{~L}$ of beer [4]. 
Currently, most of the BSG is used as animal feed, which contribute to methane gas production, while the minority are disposed in landfills [5]. Hence, other more economical and environmental friendly uses for BSG are needed.

The components of BSG consist of the barley malt grain husks, pericarp, and seed coat layers of the grains [6,7]. BSG are lignocellulosic materials that contain cellulose, non-cellulosic polysaccharides, and lignin [6]. Also, BSG were found to be abundant in proteins, essential amino acids, fiber, and phenolic compounds $[3,8]$. Nutrients such as lipids, fatty acids, and polyphenols were also found to be present in BSG $[9,10]$. The predominant lipids identified were triglyceride and the fatty acids were linoleic, palmitic, oleic, $\alpha$-linoleic, and stearic acids. Other fatty acids, including myristic and vaccenic acids, were present in lower amounts [9]. One of the main challenges in the utilization and extraction of useful components in BSG is that the proteins and nutrients are bound to the cellulose and hemicellulose. Hence, in order to extract these compounds, physical, biological, chemical pre-treatments, or a combined pre-treatment are required [11]. Biological pre-treatment would be a more environmentally friendly option as it does not require chemicals or solvents, and has the added advantage of not generating toxic compounds [12]. For agricultural by-products, solid state fermentation (SSF) commonly serves as a biological pre-treatment method, which is also convenient and economical. This is because SSF requires less energy, produces less wastewater, and hence is overall more environmentally friendly [13]. The amount of polysaccharides, along with the other nutritional contents in BSG, also makes it a suitable substrate for SSF [10]. This has been successfully carried out with fungi and some bacteria on food such as soybean waste, rice, cassava, corn cobs, and bagasse $[14,15]$. Some of the targeted products generated by SSF studies were antioxidants, protein content, and lipids [16,17]. Until now, some microorganisms, such as Trametes versicolor and Streptomyces sp. strain AMT-3, have also proven to be able to grow using BSG as the carbon and nitrogen sources for production of xylanase, laccases, and polyphenols under SSF conditions [18,19]. These studies showed the potential of using microorganisms to valorize BSG, and hence generating products that can be used in food-related processes.

Bacillus are generally regarded as safe (GRAS) species, which are well known to secrete abundant amounts of extracellular enzymes [20]. In this study, we used Bacillus subtilis (B. subtilis) WX-17, which was previously isolated from natto (traditional food in Japan, where cooked soybeans are fermented using B. subtilis, giving beneficial effects in health) [21] as the host for SSF to enrich the nutritional content of BSG. Gas chromatography-mass spectrophotometry (GC-MS) was applied for metabolomics analysis to provide important insights into the mechanism behind BSG fermentation with B. subtilis WX-17. To date, few studies have been carried out on metabolic profiling of fermented BSG. This study would help to shed light on the metabolic changes and mechanisms behind the microbial SSF of BSG. With enrichment of nutritional content in BSG, fermented BSG could possibly be used in food-related applications, such as culture medium, functional food ingredients in human diet, and value-added animal feed.

\section{Materials and Methods}

\subsection{Microorganism for Fermentation}

B. subtilis WX-17 previously isolated from Marumiya Kyushu Ichiban natto (Japan) (accession number NCIMB 15204) was maintained on nutrient agar plates at $37^{\circ} \mathrm{C}$.

\subsection{Brewer's Spent Grains and Fermentation Conditions}

BSG were taken from Asia Pacific Breweries (Singapore) Pte, Ltd. (Singapore) and stored in sealed polyethylene bags at $-80^{\circ} \mathrm{C}$ until used. B. subtilis WX-17 was cultured in sterile falcon tube with $5 \mathrm{~mL}$ of nutrient broth for $24 \mathrm{~h}$ at $37^{\circ} \mathrm{C}$.

Ten $\mathrm{g}$ of BSG was placed in boiling water for $20 \mathrm{~min}$ and cooled. The BSG were then inoculated with B. subtilis WX-17 $\left(1 \times 10^{6} \mathrm{CFU} / \mathrm{g}\right)$ and $5 \mathrm{~mL}$ of sterile water. The plates of BSG were incubated at 
$37^{\circ} \mathrm{C}$ for two days. The inoculated samples were wrapped with two layers of cling wrap. Both cling wrap layers were punctured with tiny holes using a sterile needle. Samples were collected at $0 \mathrm{~h}$ and $48 \mathrm{~h}$ to represent unfermented and fermented BSG, respectively. The collected BSG was then lyophilized and stored at $-20^{\circ} \mathrm{C}$ until further analysis.

\subsection{Gas Chromatography-Mass Spectrophotometry (GC-MS) Conditions}

Amino acids, fatty acids, carbohydrates, and tricarboxylic acid (TCA) cycle intermediates were detected via GC-MS. The tests were done by the GC-MS system (Agilent Technologies 7890A-5975C Inert MSD) equipped with a HP-5MS capillary column ( $30 \mathrm{~m} \times 0.250 \mathrm{~mm}$ i.d, $0.25 \mu \mathrm{m}$ film thickness). Samples of $1 \mu \mathrm{L}$ were injected into the system by the auto-sampler in splitless mode. Carrier gas (helium) was set to flow at $1.1 \mathrm{~mL} / \mathrm{min}$.

\subsection{Analysis of Amino Acids Using GC-MS}

Based on [22], $4 \mathrm{mg}$ of samples were re-suspended in $200 \mu \mathrm{L}$ of $6 \mathrm{M} \mathrm{HCl}$. Twenty $\mu \mathrm{L}$ of $\gamma$-aminobutyric acid $(10 \mathrm{mg} / \mathrm{mL})$ were added as the internal standard. The tubes were sealed and incubated for $12-24 \mathrm{~h}$ in an oven at $105{ }^{\circ} \mathrm{C}$. The cell hydrolysates were then dried in a heat block set at $95{ }^{\circ} \mathrm{C}$. After drying, $20 \mu \mathrm{L}$ of dimethylformamide (DMF) and $20 \mu \mathrm{L}$ of $\mathrm{N}$-tert-butyldimethylsilyl-N-methyltrifluoroacetamide with $1 \%$ tert-butyldimethylchlorosilane purchased from Sigma-Aldrich were added. The tubes were then sealed and incubated at $85^{\circ} \mathrm{C}$ for $1 \mathrm{~h}$. Samples were then centrifuged at 14,800 rpm for $5 \mathrm{~min}$ and supernatants were transferred to glass vials. Forty $\mu \mathrm{L}$ of DMF were added into the glass vials and the solution was well mixed being sent for GC-MS analysis. Each sample was measured in triplicate.

For amino acid detection, the solvent delay was set to $2.5 \mathrm{~min}$. The ion source temperature and injector temperature were set at 230 and $280^{\circ} \mathrm{C}$, respectively. The oven temperature set to hold at $160^{\circ} \mathrm{C}$ for $1 \mathrm{~min}$ then ramped to $310^{\circ} \mathrm{C}$ at the rate of $20^{\circ} \mathrm{C} / \mathrm{min}$, and finally held at $310^{\circ} \mathrm{C}$ for $0.5 \mathrm{~min}$. Data were obtained in full scan mode from 180 to $550 \mathrm{~m} / \mathrm{z}$ with a $2-4$ scan per sec. The identification of amino acids was carried out accordingly by using a NIST08 mass spectral library. Samples were normalized using $\gamma$-aminobutyric acid before comparison.

\subsection{Derivatization for Metabolomics Study}

Three $\mathrm{g}$ of fermented BSG were weighed and $10 \mathrm{~mL}$ of methanol was added. The samples were homogenized 6 times, for $30 \mathrm{~s}$ each run, using FastPrep-24 MP homogenizer. In between the homogenization, the samples were placed in ice bath to cool the sample. The samples were then centrifuged at $9000 \times g$ for $10 \mathrm{~min}$ at $4{ }^{\circ} \mathrm{C}$. The supernatant containing the metabolites, both carbohydrates, and tricarboxylic acid (TCA) cycle intermediates were pressed through a syringe attached with a $0.22 \mu \mathrm{m}$ filter. Then, $1.5 \mathrm{~mL}$ of filtered supernatant was added with $10 \mu \mathrm{L}$ of $2 \mathrm{mg} / \mathrm{mL}$ ribitol dissolved in ultrapure water as the internal standard. Samples were dried in a heat block at $30^{\circ} \mathrm{C}$ overnight. According to the method in a previous study [23], the dried samples were then derivatized with $50 \mu \mathrm{L}$ of methoxamine (MOX) hydrochloride in $20 \mathrm{mg} / \mathrm{mL}$ pyridine and incubated at $37^{\circ} \mathrm{C}$ for $1 \mathrm{~h}$. Then, silylation was carried out by adding $100 \mu \mathrm{L}$ of $\mathrm{N}$-methyl- $\mathrm{N}$-(trimethylsilyl)-trifluoroacetamide (MSTFA) with $1 \%$ trimethylchlorosilane (TMCS) to the precipitate and incubated at $70{ }^{\circ} \mathrm{C}$ for $30 \mathrm{~min}$. Each sample was measured in triplicate.

The ion source temperature and injector temperature were set at 230 and $250{ }^{\circ} \mathrm{C}$, respectively. The oven temperature was as follows: $75{ }^{\circ} \mathrm{C}$ for $4 \mathrm{~min}$, ramped to $280{ }^{\circ} \mathrm{C}$ at the rate of $4{ }^{\circ} \mathrm{C} / \mathrm{min}$, and held at $280{ }^{\circ} \mathrm{C}$ for $2 \mathrm{~min}$. Data were acquired in full scan mode from 35 to $600 \mathrm{~m} / \mathrm{z}$ with a 0.3-s scan time. The identification of carbohydrates was carried out accordingly by using the NIST08 mass spectral library. Normalization was done using ribitol before comparison. 


\subsection{Analysis of Fatty Acids Using GC-MS}

Ten mg of fermented BSG and $10 \mathrm{mg}$ of fresh BSG (control) were weighed and placed into Eppendorf tubes following a method from a previous study [23]. Then, $1 \mathrm{~mL}$ of $0.9 \% \mathrm{NaCl}$ solution and $200 \mu \mathrm{L}$ of acetic acid were added to each sample. Then, $10 \mu \mathrm{L}$ of $10 \mathrm{mg} / \mathrm{mL}$ heptadecanoic acid dissolved in ethanol was added as internal standard. The samples were sonicated for $30 \mathrm{~s}$ each. Then, $3 \mathrm{~mL}$ of a chloroform-methanol 2:1 solution was added and the samples were inverted several times, vortexed vigorously, and centrifuged at $10,000 \times g$ for $10 \mathrm{~min}$ at $4{ }^{\circ} \mathrm{C}$. Then, $1 \mathrm{~mL}$ of the chloroform layer, which is the bottom layer, was collected and dried overnight at $30^{\circ} \mathrm{C}$. The dried lipid residue was re-dissolved in $500 \mu \mathrm{L}$ BF3-methanol 10\% (FLUKA, 15716) and incubated in a sealed vial in a heat block set at $95^{\circ} \mathrm{C}$ for $20 \mathrm{~min}$. Fatty acid methyl esters (FAMEs) were extracted with the addition of $300 \mu \mathrm{L}$ saturated $\mathrm{NaCl}$ in ultrapure water, then an addition of $300 \mu \mathrm{L}$ n-hexane. Samples were vortexed for $5 \mathrm{~min}$ and centrifuged at 14,800 rpm for $5 \mathrm{~min}$. Then, $200 \mu \mathrm{L}$ of samples (top layer) were transferred into glass vials for GC-MS analysis. Each sample was measured in triplicate.

The injector temperature was set at $250^{\circ} \mathrm{C}$ and MS source temperature was set at $230^{\circ} \mathrm{C}$. The oven was held at a temperature of $80^{\circ} \mathrm{C}$ for $1 \mathrm{~min}$, then elevated to $250^{\circ} \mathrm{C}$ at a rate of $7{ }^{\circ} \mathrm{C} / \mathrm{min}$, and finally maintained at $250^{\circ} \mathrm{C}$ for $10 \mathrm{~min}$. Data were acquired in full scan from $35-600 \mathrm{~m} / \mathrm{z}$. The identification of fatty acids was carried out by using the NIST08 mass spectral library. Normalization was done by using heptadecanoic acid before comparison.

\subsection{Antioxidant Assay}

Free radical-scavenging activity of the samples was investigated by 1,1-dipheny-2-picryl-hydrazil (DPPH) with some modifications [24]. Then, $0.6 \mathrm{mM}$ of DPPH solution was prepared in ethanol. Samples for the test were prepared by adding $100 \mathrm{mg}$ of fermented BSG after 2 days to $300 \mu \mathrm{L}$ of ethanol. Then, $150 \mathrm{ul}$ of each sample was drawn and added to a solution of $250 \mu \mathrm{L}$ of ethanol and $100 \mu \mathrm{L}$ of DPPH. The tubes were then incubated in a dark place for $30 \mathrm{~min}$ at room temperature. The absorbance of each sample was measured at $515 \mathrm{~nm}$ against ethanol as the blank using Thermo Fisher NanoDrop 2000c spectrophotometer (Thermo Fisher Scientific, Wilmington, NC, USA.). The antioxidant activity was quantified in signal inhibition percentage. A standard curve was generated by Trolox standards to correlate the weight of Trolox to the signal inhibition (\%). The signal inhibition $\%$ obtained from each sample was then converted into the weight of Trolox.

\subsection{Statistical Analysis}

MetaboAnalyst 4.0 (Montreal, Canada) was utilized to construct the clustering heatmap and partial least square-discriminant analysis (PLS-DA) for statistical analysis [25]. Heatmap was clustered with calculated Euclidean distance and ward clustering algorithm. All experiments were conducted in triplicate and the standard deviation was calculated.

\section{Results}

\subsection{Analysis on Untargeted Extracellular Metabolic Profiling}

An untargeted metabolomics study was carried out using GC-MS. This was to provide insights into the changes that occurred during fermentation. A total of 35 differential metabolites were identified, which could be categorized into carbohydrates, TCA cycle intermediates, fatty acids, and amino acids. To make sense of the metabolomic changes, the metabolites were mapped on biochemical pathways. Also, to gain an overall view of the changes in metabolite abundance, analysis was carried out using a heatmap and partial least square-discriminant analysis (PLS-DA) [26]. Based on the heatmap (Figure 1), the carbohydrate levels were shown to have decreased and the amino acid, fatty acid, and TCA cycle intermediate levels were found to increase after fermentation for $48 \mathrm{hrs}$. In addition, the respective changes in the metabolites over 3 days of fermentation are shown in the heatmap in the Supplementary Materials (Figure S1). In general, after 2 days of fermentation, amino and fatty acids were upregulated, 
while carbohydrates were downregulated. However, after 3 days of fermentation, fatty acids were downregulated dramatically, which might be due to the lack of carbon source for B. subtilis WX-17 leading to the beta-oxidation of fatty acids to generate an additional carbon source. Hence, optimum valorization was achieved after 2 days of fermentation and the analyses of results were based on 2-days data, accordingly.

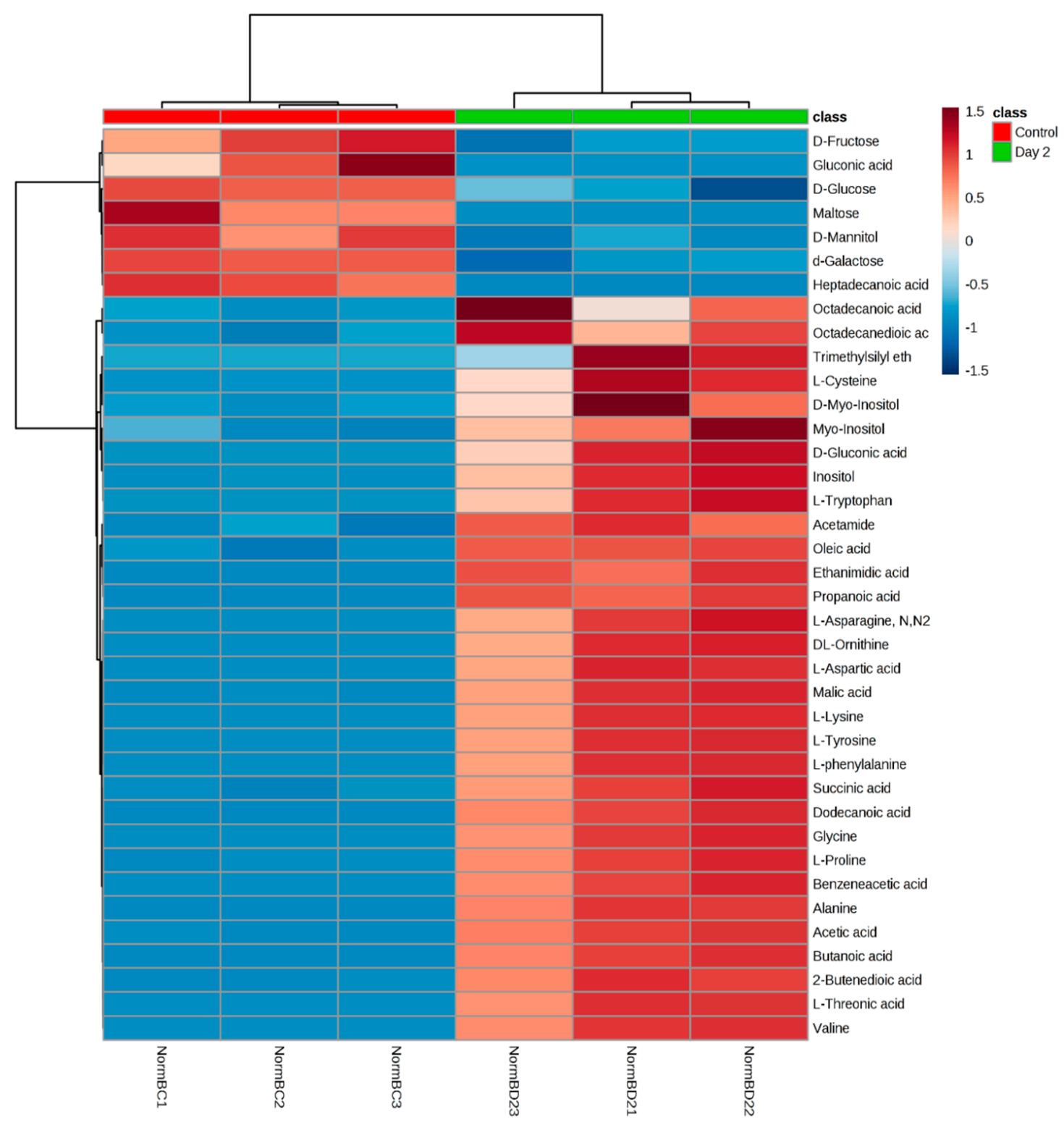

Figure 1. A heatmap analysis of metabolites variations. Fermentation was carried out for 2 days and metabolites were analyzed by gas chromatography-mass spectrophotometry (GC-MS). The unfermented brewers' spent grains (BSG) samples, in triplicate, are displayed from the left, followed by the fermented BSG samples, in triplicate. The areas shaded in red indicate a higher amount of the specific metabolites, whereas the areas shaded in blue indicate a lower amount of the specific metabolites.

Similarly, a clear difference in the metabolites between fermented and unfermented BSG was observed using PLS-DA, as shown in Figure 2. The principal components, PC1 and PC2, displayed a $95.3 \%$ and $3.3 \%$ variance, respectively. The PLS-DA plot had a variance $R^{2}$ value of 0.99 , which is considered to be extremely substantial [27]. The observed trend of having overall significant changes in the metabolites based on PLS-DA analysis was congruent and correlated with the trend observed from the clustering heatmap. The metabolic profiles were further investigated by the use of 
respective component tests and biochemical pathways triggered during the fermentation of BSG with B. subtilis WX-17.

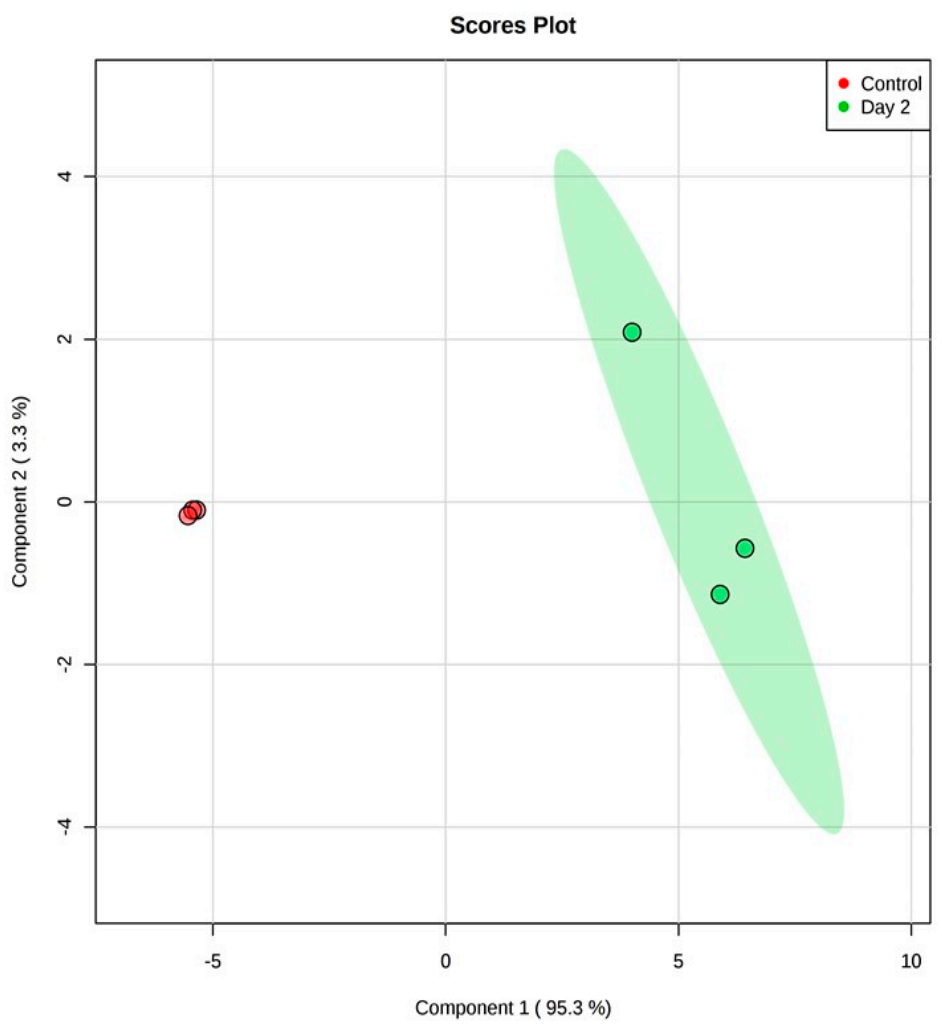

Figure 2. A partial least square-discriminant analysis (PLS-DA) score plot for metabolites analyzed by GC-MS for unfermented BSG (red) and fermented BSG samples (green), with a 95\% confidence interval. Each dot represents all of the metabolites detected in each replicate.

\subsection{Amino Acids Analysis}

The amino acid metabolites were found to increase in the fermented BSG sample as compared to unfermented BSG (Table 1). Several amino acids metabolites were detected, including leucine, phenylalanine, lysine, threonine, serine, proline, glutamic acid, aspartic acid, and tyrosine. The increases in the respective amino acids were calculated and expressed as fold changes (Table 1). Among the amino acids, proline was found to have the highest increase, at 3.5 times. In terms of total amino acids, the amount increased from $0.859 \mathrm{mg} / \mathrm{g}$ in unfermented BSG to $1.894 \mathrm{mg} / \mathrm{g}$ in fermented BSG after 2 days of fermentation.

Table 1. Amino acids results $(\mathrm{mg} / \mathrm{g})$ before and after fermentation.

\begin{tabular}{cccc}
\hline Amino Acids & $\begin{array}{c}\text { Unfermented Brewers' } \\
\text { Spent Grains (BSG) }\end{array}$ & $\begin{array}{c}\text { Fermented Brewers' Spent } \\
\text { Grains (BSG) (Day 2) }\end{array}$ & Fold Change \\
\hline Leucine & $0.113 \pm 0.031$ & $0.134 \pm 0.098$ & 1.185 \\
Serine & $0.015 \pm 0.005$ & $0.017 \pm 0.009$ & 1.812 \\
Aspartic Acid & $0.024 \pm 0.003$ & $0.034 \pm 0.007$ & 1.542 \\
Threonine & $0.015 \pm 0.005$ & $0.026 \pm 0.016$ & 2.092 \\
Phenylalanine & $0.021 \pm 0.003$ & $0.027 \pm 0.015$ & 2.133 \\
Proline & $0.349 \pm 0.182$ & $1.230 \pm 0.568$ & 3.527 \\
Glutamic Acid & $0.304 \pm 0.20$ & $0.407 \pm 0.376$ & 1.625 \\
Lysine & $0.012 \pm 0.006$ & $0.011 \pm 0.010$ & 1.200 \\
Tyrosine & $0.006 \pm 0.001$ & $0.009 \pm 0.001$ & 1.560 \\
Total amino acids & $0.859 \pm 0.049$ & $1.894 \pm 0.125$ & 2.204 \\
\hline
\end{tabular}




\subsection{Fatty Acids Analysis}

It was shown that the total fatty acid content of BSG increased in the fermented BSG sample as compared to unfermented BSG sample (Table 2). A total of four fatty acids were detected by GC-MS analysis. The fatty acids were hexadecenoic acid (palmitic acid), 9,12-octadecanoic acid (linoleic acid), 9-Octadecanoic acid (oleic acid), and octadecanoic acid (stearic acid). These could be categorized into saturated or unsaturated fatty acids. The essential unsaturated fatty acids were linoleic acid and oleic acid, whereas palmitic acid and stearic acid were the saturated fatty acids. Oleic acid was found to have the highest increase with a 2.366-fold increase, whereas palmitic acid was found to decrease by 0.844 -fold in the fermented BSG as compared to unfermented BSG (Table 2). Overall, increasing levels of essential unsaturated fatty acids, and decreasing or unchanged levels of saturated fatty acids after 2 days of fermentation, were observed. Unsaturated fatty acids, such as linoleic acid or oleic acid, would slightly increase the level high density lipoprotein (HDL) cholesterol, also known as good cholesterol, in humans. HDL is reported to aid in the removal of triacylglycerols from the bloodstream. Hence, the increase in unsaturated fatty acids could be interpreted as a higher nutritional value in fermented BSG [28].

Table 2. Fatty acids results (mg/g) in BSG before and after fermentation.

\begin{tabular}{cccc}
\hline Fatty Acids & Unfermented BSG & Fermented BSG (Day 2) & Fold Change \\
\hline Palmitic Acid & $1.805 \pm 0.003$ & $1.523 \pm 0.205$ & 0.844 \\
Linoleic Acid & $0.445 \pm 0.100$ & $0.731 \pm 0.220$ & 1.643 \\
Oleic Acid & $0.041 \pm 0.006$ & $0.097 \pm 0.053$ & 2.366 \\
Stearic Acid & $4.596 \pm 0.091$ & $4.734 \pm 0.131$ & 1.03 \\
Total Fatty Acids & $6.89 \pm 0.055$ & $7.085 \pm 0.152$ & 1.028 \\
\hline
\end{tabular}

\subsection{Carbohydrates Analysis}

In total, 5 carbohydrates were detected. The carbohydrates detected from GC-MS were D-fructose, D-mannitol, D-glucose, D-galactose, and maltose. After 2 days of fermentation, it was observed that D-fructose, D-mannitol, D-glucose, D-galactose, and maltose decreased by $64.9 \%, 68.9 \%, 62.2 \%, 66.5 \%$, and $86.2 \%$, respectively (Figure 3 ).

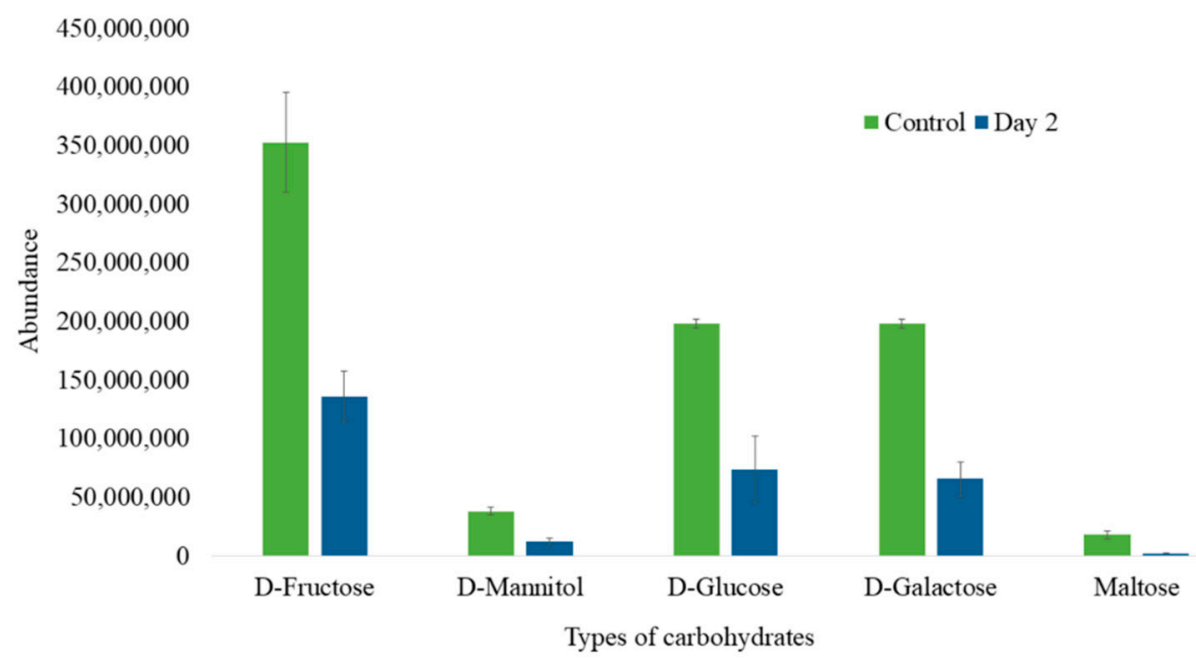

Figure 3. Metabolites belonging to the class of carbohydrate, as analyzed by GC-MS. Green bars represent the levels of the unfermented BSG samples, whereas the blue bars represent the levels of the fermented BSG samples. 


\subsection{Antioxidant Test}

The antioxidant properties of the samples were evaluated through DPPH radical scavenging activity and quantified by Trolox equivalent (Table 3). The method used DPPH assay, which is a test of the amount of electron donation of antioxidants to neutralize the deep purple DPPH radical. The respective samples displayed a visual discoloration, which is reflected in the signal inhibition $\%$. The conversion of signal inhibition \% to weight of Trolox was done via calibration curve and it was observed that fermented BSG showed an increase in Trolox equivalent from $1.2 \pm 0.03 \mu \mathrm{g} / \mathrm{g}$ BSG to $6.94 \pm 0.21 \mu \mathrm{g} / \mathrm{g}$ BSG, an approximate 5.8-times increase in antioxidant activity.

Table 3. Trolox quantification for antioxidant activity before and after fermentation.

\begin{tabular}{ccc}
\hline Samples & Signal Inhibition \% & Weight of Trolox $(\mu \mathrm{g} / \mathrm{g}$ BSG) \\
\hline Unfermented BSG (Control) & 4.72 & $1.2 \pm 0.03$ \\
Fermented BSG (Day 2) & 28.27 & $6.94 \pm 0.21$ \\
\hline
\end{tabular}

\section{Discussion}

The regulations in the metabolites after fermentation were hypothesized by analyzing them using various biochemical pathways. The respective biochemical pathways could have possibly triggered the changes in the metabolites during the fermentation.

\subsection{Carbohydrate Pathway Analysis}

In the carbohydrate analysis, it was observed that D-fructose, D-mannitol, D-glucose, D-galactose, and maltose obviously decreased after fermentation (Figure 3). This result indicates that the various sugars were being used by $B$. subtilis WX-17 for growth. It is speculated that various microbial enzymes, such as invertase, amylase, cellulose, and pectinase, are first produced as a bacterial fermentation progress to hydrolyze long-chain polymeric sugars and starch molecules into simpler carbohydrates during fermentation by B. subtilis WX-17 [29-32]. For the inter-conversion among the carbohydrates, maltose can be catalyzed by maltose- 6 '-phosphate glucosidase to produce D-glucose (starch and sucrose metabolism pathway). D-glucose can be converted into alpha-D-glucose-1P and then converted into uridine diphosphate (UDP-glucose) with the aid of the enzyme, UDP glucose pyrophosphorylase [33]. UDP-glucose produces sucrose, which in turn is converted into fructose through the enzyme sucrose phosphorylase (starch and sucrose metabolism); fructose can be converted back into D-glucose or alpha-D-glucose to produce galactose (galactose metabolism pathway). In the following step, glycolysis converts glucose into pyruvate to supply adenosine triphosphate (ATP) and nicotinamide adenine dinucleotide (NADH), which triggers the generation of other primary or secondary metabolites that are important to cell growth. Besides, it has been reported that BSG contains phytic acid and fermentation reduces the level of phytic acid [7]. This leads to an increase in the product myo-inositol from phytic acid hydrolysis. Myo-inositol was also detected to have increased based on the GC-MS analysis, which reflected the reduction of the antinutrient phytic acid.

\subsection{Amino Acids Metabolism Analysis}

It was observed that the amount of amino acids increased in the fermented BSG sample, as compared to unfermented BSG. This could be attributed to the enzymes, such as proteases, which are produced by B. subtilis. This hydrolyzes the complex proteins present in BSG into simple amino acids [34]. The production of proteases from microorganisms for proteolysis during fermentation and release of amino acids was also observed in another fermented food study [35]. In addition, it is also possible that the amino acids were synthesized from, or associated with, the carbohydrate metabolic pathways.

For instance, serine can be synthesized through glycolysis. In turn, this can aid the synthesis of glycine and threonine, as reactions are reversible. The series of correlated serine, glycine, and threonine 
pathways can be seen in Figure 4. The production of serine yields cysteine (cysteine and methionine metabolism pathway). The reversible reactions in the alanine pathway produce pyruvate, valine, and leucine. Mainly, glycolysis and breakdown of amino acids contribute to the production of pyruvate. A decrease in the precursors for glycolysis, such as D-fructose and D-mannitol, could possibly be due to the upregulation of glycolysis. The upregulation of glycolysis increased the production of amino acids (Figure 4). The product of glycolysis, pyruvate, is then converted into acetyl-CoA, which enters the TCA cycle to produce energy, and also aspartic acid. Valine, alanine, and asparagine are produced by aspartic acid (alanine, aspartate, and glutamate metabolism pathway). Also, lysine can be produced from aspartic acid (lysine biosynthesis pathway). Lysine is also produced from 2-oxoglutarate, which is an intermediate of the TCA cycle. The increasing amount of intermediate after fermentation drives the metabolic flux towards the production of lysine, which explains its increase. Glutamate can also be produced when pyruvate enters the TCA cycle. Proline is produced from glutamate (alanine, aspartate, and glutamate metabolism pathway). Proline can also be produced by arginine (arginine and proline metabolism pathway). Through the urea cycle, aspartic acid can be converted into arginine. The production of phenylalanine, tyrosine, and tyroptophan mainly stems from the compound phosphoenolpyruvate (PEP). PEP is converted into shikimate with the aid of the enzyme shikimate dehydrogenase, which then forms chorismate (shikimate pathway). Chorismate synthesizes either tryptophan or L-arogenate to produce phenylalanine and tyrosine (phenylalanine, tyrosine, and tryptophan biosynthesis pathway). Most of the amino acids identified were found to be glucogenic amino acids. Such amino acids could be converted into glucose. On the other hand, a few exclusively ketogenic amino acids, including lysine and leucine, were detected as well.



Figure 4. Metabolic changes mapped on metabolic pathways during fermentation of BSG by B. Subtilis WX-17. Colored labels indicate the respective increase, decrease, or undetected levels of metabolites at each given time.

Inferring from the results and hypothesized pathway analyses, SSF using B. subtilis WX-17 on BSG as a substrate was able to utilize the respective useful components in BSG. The increase in production of amino acids is similar to the observation in another SSF study of cassava using Trichoderma pseudokoningii, where protein content was increased from $8.4 \%$ to $12.5 \%$ [36]. It could be deduced that SSF produced useful enzymes and compounds that improved the nutritional content of the food by-product. The increase in amino acid production from the results was also in-line with an increase in amino acids observed in SSF of soybeans with B. subtilis [37]. In terms of amino acids 
production, SSF has been evaluated on different food by-products and results have shown that it leads to an upregulation of amino acids.

\subsection{TCA Cycle Metabolism Analysis}

The TCA cycle is an important sequence of enzyme-catalyzed reactions that aerobic microorganisms use to generate energy. Metabolites belonging to the TCA cycle were found to increase in the fermented BSG sample, as compared to unfermented BSG. As fermentation progresses, the synthesis of intermediates in the TCA cycle increase as well [38]. Based on the upregulation of glycolysis, this leads to an increase in pyruvate, which is then converted into acetyl coenzyme A (acetyl-CoA) and enters the TCA cycle. The increased amounts of precursor, acetyl-CoA, into the TCA cycle subsequently leads to an increase in the TCA cycle intermediates (Figure 4). The analysis showed increased levels of succinic acid and malic acid, which are key components in the TCA cycle. The synthesis of these components are catalyzed by the enzymes succinate dehydrogenase and malate dehydrogenase, respectively [33].

\subsection{Fatty Acid Metabolism Analysis}

Fatty acids were suggested to be produced from acetyl-CoA and facilitated by the enzyme fatty acid synthase (fatty acid biosynthesis pathway). Also, B. subtilis WX-17 could have possibly produced lipases, which help to hydrolyze lipids present in BSG into fatty acids $[39,40]$. In the presence of fatty acid synthase, various reactions are catalyzed and saturated fatty acids, such as stearic acid and palmitic acid, are produced through elongation and hydrolysis. Unsaturation would cause the saturated fatty acids to be converted into unsaturated fatty acids, such as oleic acid and linoleic acid. With the increase in the precursor acetyl-CoA due to upregulation of glycolysis observed after fermentation, it was postulated that saturated fatty acids would increase. However, this was not the case, as saturated fatty acids were downregulated, while unsaturated fatty acids were upregulated. This is possibly due to the rate of unsaturation being higher than the rate of production of unsaturated fatty acids. As can be seen from the results, the results after fermentation gave an increase of 1.643 and 2.366 times for unsaturated fatty acids, which are linoleic acid, and oleic acid, respectively. The increase in the fatty acids trend was also observed in another study with soybean fermentation using B. subtilis, where increase in unsaturated fatty acids and decrease in saturated fatty acids were observed [41]. Also, the results and pathway analysis suggested a similar trend compared to a study that worked on SSF of rice bran using Mucor rouxiil, which showed an increase in unsaturated fatty acids, such as gamma-linolenic acid [42]. Overall, microbial SSF across these food by-products have displayed a trend of increased unsaturated fatty acids.

\subsection{Antioxidant Test Analysis}

BSG has been reported to be a source of antioxidant phenolic compounds, which could be present in the husk and cell walls [43,44]. Particularly for phenolic acids content in BSG, ferulic acid and $p$-coumaric acid were found to be present in relatively high concentrations [43]. Other phenolic compounds present include flavonoids, proanthocyanidins, and amino phenolic compounds. There are various methods such as solid to liquid extraction, acid hydrolysis, and saponification to extract phenolic compounds from BSG fermentation [43,44]. It was reported that bioactive phenolic compounds can also be successfully extracted from natural sources using solid state fermentation [45]. In particular, it has been reported that fermentation with $B$. subtilis could produce nattokinase, which is a polypetide that has antioxidant activity $[46,47]$. Hence, both solid state fermentation process and production of nattokinase could account for the increase in antioxidant properties and phenolic content in fermented BSG shown in the results.

\section{Conclusions}

This work demonstrated valorizing BSG through solid state bacterial fermentation using B. subtilis WX-17. The increased amount of amino acids, unsaturated fatty acids, and antioxidants after 
fermentation showed the capabilities of B. Subtilis WX-17 to degrade the complex macronutrients in BSG into useful components. The increase in the respective useful components suggested that microbial SSF on BSG were able to produce other useful compounds in addition to the other products from SSF, such as the enzymes and polyphenols from other studies. The in-depth investigation using GC-MS, statistical analysis, and pathway analysis provided the metabolic changes at different time points during fermentation. With the enhanced nutritional content of fermented BSG, future work could involve food-related applications or examine more novel fermentation methods for effective valorization of BSG.

Supplementary Materials: Supplementary materials can be found at http://www.mdpi.com/2311-5637/5/3/52/s1.

Author Contributions: Y.X.T. and W.K.M. performed the experiments and wrote the manuscript. J.L. commented on the experiment and manuscript. K.J. provided technical assistance. W.N.C. reviewed and edited the manuscript. All authors read and approved the final manuscript.

Funding: The funding of this research was supported by the Interdisciplinary Graduate School at Nanyang Technological University. Funding was also from Zhong Feng International (M4062121.120.703012).

Acknowledgments: The authors would like to thank the Nayang Environment and Water Research Institute (NEWRI), Singapore, and the Interdisciplinary Graduate School (IGS), Nayang Technological University, Singapore, for the award of a research scholarship to Tan Yong Xing and the support for this research.

Conflicts of Interest: The authors declare no conflict of interest. The funders had no role in the design of the study; in the collection, analyses, or interpretation of data; in the writing of the manuscript, or in the decision to publish the results.

\section{References}

1. Waqas, M.; Rehan, M.; Khan, M.D.; Nizami, A.-S. Conversion of Food Waste to Fermentation Products. In Encyclopedia of Food Security and Sustainability; Ferranti, P., Berry, E.M., Anderson, J.R., Eds.; Elsevier: Amsterdam, The Netherlands, 2019; pp. 501-509.

2. Mussatto, S.I. Biotechnological Potential of Brewing Industry By-Products. In Biotechnology for Agro-Industrial Residues Utilisation: Utilisation of Agro-Residues; Singh nee' Nigam, P., Pandey, A., Eds.; Springer: Berlin/Heidelberg, Germany, 2009; pp. 313-326.

3. Mussatto, S.I. Brewer's spent grain: A valuable feedstock for industrial applications. J. Sci. Food Agric. 2014, 94, 1264-1275. [CrossRef] [PubMed]

4. Mathias, T.R.D.S.; Fernandes de Aguiar, P.; de Almeida e Silva, J.B.; Moretzsohn de Mello, P.P.; Camporese Sérvulo, E.F. Brewery Waste Reuse for Protease Production by Lactic Acid Fermentation. Food Technol. Biotechnol. 2017, 55, 218-224. [CrossRef] [PubMed]

5. Kerby, C.; Vriesekoop, F. An Overview of the Utilisation of Brewery By-Products as Generated by British Craft Breweries. Beverages 2017, 3, 24. [CrossRef]

6. Mussatto, S.I.; Dragone, G.; Roberto, I.C. Brewers' spent grain: Generation, characteristics and potential applications. J. Cereal Sci. 2006, 43, 1-14. [CrossRef]

7. Lynch, K.M.; Steffen, E.J.; Arendt, E.K. Brewers' spent grain: A review with an emphasis on food and health. J. Inst. Brew. 2016, 122, 553-568. [CrossRef]

8. Ikram, S.; Huang, L.; Zhang, H.; Wang, J.; Yin, M. Composition and Nutrient Value Proposition of Brewers Spent Grain. J. Food Sci. 2017, 82, 2232-2242. [CrossRef]

9. Fărcaş, A.C.; Socaci, S.A.; Dulf, F.V.; Tofană, M.; Mudura, E.; Diaconeasa, Z. Volatile profile, fatty acids composition and total phenolics content of brewers' spent grain by-product with potential use in the development of new functional foods. J. Cereal Sci. 2015, 64, 34-42. [CrossRef]

10. Salihu, A.; Bala, M. Brewer's spent grain: A review of its potentials and applications. Afr. J. Biotechnol. 2011, 10, 324-331. [CrossRef]

11. Behera, S.; Arora, R.; Nandhagopal, N.; Kumar, S. Importance of chemical pretreatment for bioconversion of lignocellulosic biomass. Renew. Sustain. Energy Rev. 2014, 36, 91-106. [CrossRef]

12. Sindhu, R.; Binod, P.; Pandey, A. Biological pretreatment of lignocellulosic biomass-An overview. Bioresour. Technol. 2016, 199, 76-82. [CrossRef]

13. Pandey, A. Solid-state fermentation. Biochem. Eng. J. 2003, 13, 81-84. [CrossRef] 
14. Couto, S.R.; Sanromán, M.Á. Application of solid-state fermentation to food industry-A review. J. Food Eng. 2006, 76, 291-302. [CrossRef]

15. Madamwar, D.; Patel, S.; Parikh, H. Solid state fermentation for cellulases and $\beta$-glucosidase production by Aspergillus niger. J. Ferment. Bioeng. 1989, 67, 424-426. [CrossRef]

16. Queiroz Santos, V.A.; Nascimento, C.G.; Schmidt, C.A.P.; Mantovani, D.; Dekker, R.F.H.; da Cunha, M.A.A. Solid-state fermentation of soybean okara: Isoflavones biotransformation, antioxidant activity and enhancement of nutritional quality. LWT 2018, 92, 509-515. [CrossRef]

17. Lizardi-Jimenez, M.A.; Hernandez-Martinez, R. Solid state fermentation (SSF): Diversity of applications to valorize waste and biomass. 3 Biotech 2017, 7, 44. [CrossRef] [PubMed]

18. Tišma, M.; Jurić, A.; Bucić-Kojić, A.; Panjičko, M.; Planinić, M. Biovalorization of brewers' spent grain for the production of laccase and polyphenols. J. Inst. Brew. 2018, 124, 182-186. [CrossRef]

19. Nascimento, R.P.; Coelho, R.R.R.; Marques, S.; Alves, L.; Gírio, F.M.; Bon, E.P.S.; Amaral-Collaço, M.T. Production and partial characterisation of xylanase from Streptomyces sp. strain AMT-3 isolated from Brazilian cerrado soil. Enzym. Microb. Technol. 2002, 31, 549-555. [CrossRef]

20. Schallmey, M.; Singh, A.; Ward, O.P. Developments in the use of Bacillus species for industrial production. Can. J. Microbiol. 2004, 50, 1-17. [CrossRef]

21. Nishinari, K.; Fang, Y.; Nagano, T.; Guo, S.; Wang, R. 6-Soy as a food ingredient. In Proteins in Food Processing, 2nd ed.; Yada, R.Y., Ed.; Woodhead Publishing: Cambridge, UK, 2018; pp. 149-186.

22. Zamboni, N.; Fendt, S.-M.; Rühl, M.; Sauer, U. 13C-based metabolic flux analysis. Nat. Protoc. $2009,4,878$. [CrossRef]

23. Chen, L.; Chen, W.N. Metabolite and Fatty Acid Analysis of Yeast Cells and Culture Supernatants. Bio-protocol 2014, 4, e1219. [CrossRef]

24. Wan, C.; Yu, Y.; Zhou, S.; Liu, W.; Tian, S.; Cao, S. Antioxidant activity and free radical-scavenging capacity of Gynura divaricata leaf extracts at different temperatures. Pharmacogn. Mag. 2011, 7, 40-45. [CrossRef] [PubMed]

25. Chong, J.; Soufan, O.; Li, C.; Caraus, I.; Li, S.; Bourque, G.; Wishart, D.S.; Xia, J. MetaboAnalyst 4.0: Towards more transparent and integrative metabolomics analysis. Nucleic Acids Res. 2018, 46, W486-W494. [CrossRef] [PubMed]

26. Gromski, P.S.; Muhamadali, H.; Ellis, D.I.; Xu, Y.; Correa, E.; Turner, M.L.; Goodacre, R. A tutorial review: Metabolomics and partial least squares-discriminant analysis-a marriage of convenience or a shotgun wedding. Anal. Chim. Acta 2015, 879, 10-23. [CrossRef] [PubMed]

27. Henseler, J.; Ringle, C.M.; Sinkovics, R.R. The use of partial least squares path modeling in international marketing. In New Challenges to International Marketing; Emerald Group Publishing Limited: Bingley, UK, 2009; Volume 20, pp. 277-319.

28. Lunn, J.; Theobald, H.E. The health effects of dietary unsaturated fatty acids. Nutr. Bull. 2006, 31, 178-224. [CrossRef]

29. Lincoln, L.; More, S.S. Bacterial invertases: Occurrence, production, biochemical characterization, and significance of transfructosylation. J. Basic Microbiol. 2017, 57, 803-813. [CrossRef] [PubMed]

30. Raul, D.; Biswas, T.; Mukhopadhyay, S.; Kumar Das, S.; Gupta, S. Production and Partial Purification of Alpha Amylase from Bacillus subtilis (MTCC 121) Using Solid State Fermentation. Biochem. Res. Int. 2014, $2014,5$. [CrossRef]

31. Sethi, S.; Datta, A.; Gupta, B.L.; Gupta, S. Optimization of Cellulase Production from Bacteria Isolated from Soil. Isrn Biotechnol. 2013, 2013, 7. [CrossRef]

32. Ahlawat, S.; Dhiman, S.S.; Battan, B.; Mandhan, R.P.; Sharma, J. Pectinase production by Bacillus subtilis and its potential application in biopreparation of cotton and micropoly fabric. Process Biochem. 2009, 44, 521-526. [CrossRef]

33. Kanehisa, M.; Furumichi, M.; Tanabe, M.; Sato, Y.; Morishima, K. KEGG: New perspectives on genomes, pathways, diseases and drugs. Nucleic Acids Res. 2016, 45. [CrossRef]

34. Oyeleke, S.B.; Oyewole, O.; Egwim, E. Production of Protease and Amylase from Bacillus subtilis and Aspergillus niger Using Parkia biglobossa (Africa Locust Beans) as Substrate in Solid State Fermentation. Adv. Life Sci. 2012, 1, 49-53. [CrossRef] 
35. Lee, D.E.; Shin, G.R.; Lee, S.; Jang, E.S.; Shin, H.W.; Moon, B.S.; Lee, C.H. Metabolomics reveal that amino acids are the main contributors to antioxidant activity in wheat and rice gochujangs (Korean fermented red pepper paste). Food Res. Int. (Ott. Ont.) 2016, 87, 10-17. [CrossRef] [PubMed]

36. Bayitse, R.; Hou, X.; Laryea, G.; Bjerre, A.-B. Protein enrichment of cassava residue using Trichoderma pseudokoningii (ATCC 26801). AMB Express 2015, 5, 80. [CrossRef] [PubMed]

37. Sarkar, P.K.; Jones, L.J.; Craven, G.S.; Somerset, S.M.; Palmer, C. Amino acid profiles of kinema, a soybean-fermented food. Food Chem. 1997, 59, 69-75. [CrossRef]

38. Song, H.; Lee, S.Y. Production of succinic acid by bacterial fermentation. Enzym. Microb. Technol. 2006, 39, 352-361. [CrossRef]

39. Suci, M.; Arbianti, R.; Hermansyah, H. Lipase production from Bacillus subtilis with submerged fermentation using waste cooking oil. In Proceedings of the IOP Conference Series: Earth and Environmental Science, Bali, Indonesia, 3 October 2017.

40. Lesuisse, E.; Schanck, K.; Colson, C. Purification and preliminary characterization of the extracellular lipase of Bacillus subtilis 168, an extremely basic pH-tolerant enzyme. Eur. J. Biochem. 1993, 216, 155-160. [CrossRef] [PubMed]

41. Kanghae, A.; Eungwanichayapant, P.D.; Chukeatirote, E. Fatty acid profiles of fermented soybean prepared by Bacillus subtilis and Rhizopus oligosporus. Environ. Exp. Biol. 2017, 15, 173-176. [CrossRef]

42. Jangbua, P.; Laoteng, K.; Kitsubun, P.; Nopharatana, M.; Tongta, A. Gamma-linolenic acid production of Mucor rouxii by solid-state fermentation using agricultural by-products. Lett. Appl. Microbiol. 2009, 49, 91-97. [CrossRef]

43. McCarthy, A.; O'Callaghan, Y.; Piggott, C.; FitzGerald, R.; O’Brien, N.M. Brewers' spent grain; Bioactivity of phenolic component, its role in animal nutrition and potential for incorporation in functional foods: A review. Proc. Nutr. Soc. 2012, 72, 1-9. [CrossRef]

44. Meneses, N.G.T.; Martins, S.; Teixeira, J.A.; Mussatto, S.I. Influence of extraction solvents on the recovery of antioxidant phenolic compounds from brewer's spent grains. Sep. Purif. Technol. 2013, 108, 152-158. [CrossRef]

45. Martins, S.; Mussatto, S.I.; Martínez-Avila, G.; Montañez-Saenz, J.; Aguilar, C.N.; Teixeira, J.A. Bioactive phenolic compounds: Production and extraction by solid-state fermentation. A review. Biotechnol. Adv. 2011, 29, 365-373. [CrossRef]

46. Weng, Y.; Yao, J.; Sparks, S.; Wang, K.Y. Nattokinase: An Oral Antithrombotic Agent for the Prevention of Cardiovascular Disease. Int. J. Mol. Sci. 2017, 18, 523. [CrossRef] [PubMed]

47. Mani, V.; Ming, L.C. Chapter 19-Tempeh and Other Fermented Soybean Products Rich in Isoflavones. In Fermented Foods in Health and Disease Prevention; Frias, J., Martinez-Villaluenga, C., Peñas, E., Eds.; Academic Press: Cambridge, MA, USA, 2017; pp. 453-474. 